\title{
Participación de los sistemas endógenos de péptidos opioides en los mecanismos de reforzamiento y dependencia al alcohol
}

\author{
Milagros Méndez ${ }^{1}$
}

Artículo original

\section{SUMMARY}

Biochemical and behavioral evidence indicates that the dopaminergic mesolimbic system plays a key role in the mechanisms of reinforcement and reward elicited by alcohol (ethanol) and other drugs of abuse. In addition, the dopaminergic activity of the nigrostriatal pathway has been proposed to determine brain sensitivity to ethanol, a process which could be associated to drug addiction. Besides dopamine, several neurotransmitters and neuromodulators are involved in ethanol reinforcement, including gamma aminobutyric acid (GABA), glutamate, serotonin, acetylcholine and opioid peptides (enkephalins, endorphins and dynorphins). Ethanol and opioids share several pharmacological properties and exhibit similar behavioral effects in animals and humans. These and other studies suggest that the alcohol reinforcing properties are due, at least in part, to the ethanol-induced activation of endogenous opioidergic systems. This activation could in turn increase the hedonic value and the reinforcing effects of the drug. Thus, ethanol-induced changes in opioidergic transmission could contribute to alcohol intoxication and to the neuroadaptive responses produced by the long-lasting exposure to the drug. Opioidergic transmission may be altered by ethanol at different levels, including biosynthesis, release and inactivation of opioid peptides, as well as binding of endogenous opioids to their receptors. Several studies suggest that mu and delta opioid receptors play a key role in ethanol reinforcement and dependence. Therefore, enkephalins and $\beta$-endorphin could mediate ethanol actions in the brain and play a major role in high alcohol drinking behavior. During the last years, our research group has focused on the role of the endogenous opioid systems in these processes. Evidence obtained in our laboratory suggests that enkephalins and $\beta$-endorphin differentially and selectively participate in ethanol reinforcement and dependence.

Key words: Alcohol (ethanol), reinforcement, dependence, endogenous opioid systems, enkephalins, $\beta$-endorphin, mesocorticolimbic system, nigrostriatal pathway.

\section{RESUMEN}

Evidencias bioquímicas y conductuales indican que el sistema dopaminérgico mesolímbico cumple un papel fundamental en los mecanismos de reforzamiento y recompensa del alcohol (etanol) y otras drogas de abuso. Se ha propuesto también que la actividad de la vía dopaminérgica nigroestriatal determina la sensibilidad cerebral a etanol, lo que parece estar directamente relacionado con los procesos de adicción a la droga. Además de la dopamina, varios neurotransmisores y neuromoduladores están implicados en los mecanismos de reforzamiento del etanol, entre ellos, el ácido gama-aminobutírico (GABA), el glutamato, la serotonina, la acetilcolina y los péptidos opioides (encefalinas, endorfinas y dinorfinas). El alcohol y los opioides comparten características farmacológicas y exhiben efectos similares sobre el comportamiento en animales y en el hombre. Éstos y otros estudios sugieren que las propiedades reforzadoras del etanol se deben, al menos parcialmente, a la activación de los sistemas endógenos de péptidos opioides, proceso que es inducido por el propio alcohol. Esta activación podría, a su vez, aumentar el valor hedónico y los efectos reforzadores de la droga. Los cambios inducidos por etanol sobre la transmisión de opioides podrían contribuir de manera importante a los procesos de intoxicación y a las respuestas neuronales adaptativas que produce el consumo prolongado de la droga. La transmisión opioidérgica puede ser afectada por etanol a distintos niveles, incluyendo la biosíntesis, liberación e inactivación de los opioides endógenos, así como la unión de éstos a sus receptores. Numerosas evidencias sugieren que los receptores opioides mu y delta desempeñan un papel fundamental en el reforzamiento y la dependencia al etanol. Así, las encefalinas y la $\beta$-endorfina actuarían como mediadores fisiológicos de las acciones del etanol en el cerebro, desempeñando un papel crucial en las conductas de alto consumo de la droga. En los últimos años, nuestro grupo se ha centrado en investigar el papel de los sistemas endógenos de péptidos opioides en estos procesos. Las evidencias obtenidas en nuestro laboratorio sugieren que las encefalinas y la $\beta$-endorfina participan en forma diferencial $y$ selectiva en el reforzamiento y la dependencia al etanol.

Palabras clave: Alcohol (etanol), reforzamiento, dependencia, sistemas opioides endógenos, encefalinas, $\beta$-endorfina, sistema mesocorticolímbico, vía nigroestriatal.

Departamento de Neuroquímica, Subdirección de Investigaciones Clínicas, Instituto Nacional de Psiquiatría Ramón de la Fuente Muñiz.

Correspondencia: Dra. Milagros Méndez. Departamento de Neuroquímica, Subdirección de Investigaciones Clínicas, Instituto Nacional de Psiquiatría Ramón de la Fuente Muñiz. Calz. México-Xochimilco 101, San Lorenzo Huipulco, Tlalpan, 14370, México DF. Tel. 4160-5091. Fax. 4160-5420. E-mail: ubach@imp.edu.mx 


\section{INTRODUCCIÓN}

El alcoholismo es uno de los principales problemas de salud en el mundo y el alcohol es una de las drogas más ampliamente utilizadas. En México, el alcoholismo se asocia a por lo menos cinco de las siete principales causas de mortalidad en el país ${ }^{1}$ y $25.4 \%$ de los mexicanos entre 18 y 65 años de edad abusa y/o presenta dependencia al alcohol. ${ }^{2}$ Dada la alta prevalencia de este trastorno en nuestro país, y ya que el alcohol es una droga fuertemente adictiva, es fundamental conocer los mecanismos de acción del alcohol en el cerebro, tanto a nivel de las propiedades reforzadoras sobre vías neuronales específicas como a nivel de los cambios neuroadaptativos que produce el consumo prolongado de la droga en el cerebro. El conocimiento de estos mecanismos contribuiría de manera importante al diseño de nuevas estrategias terapéuticas en el tratamiento del alcoholismo.

En los últimos años, nuestro grupo se ha centrado en investigar los mecanismos de acción del alcohol sobre vías cerebrales que forman parte de los circuitos de reforzamiento y recompensa de drogas de abuso (i.e., vía mesocorticolímbica). En particular, nuestro interés es investigar la participación de los sistemas endógenos de péptidos opioides en los mecanismos de reforzamiento y dependencia al alcohol. Para ello, diseñamos varios paradigmas experimentales que nos han permitido estudiar cómo se modifica la transmisión de encefalinas y $\beta$-endorfina en respuesta a la exposición aguda y crónica de etanol, particularmente en áreas cerebrales de la vía mesocorticolímbica. Además, hemos correlacionado los cambios neuroquímicos inducidos por etanol con los efectos conductuales producidos por la droga. Se revisan a continuación algunos de los hallazgos más importantes de nuestras investigaciones.

\section{ANTECEDENTES}

El alcohol es una droga depresora del Sistema Nervioso Central (SNC) que afecta numerosas funciones en el organismo, incluyendo la regulación de la temperatura, la coordinación motora y los patrones de sueño. Sin embargo, los efectos del alcohol en el SNC dependen de la dosis administrada, particularmente a nivel conductual. El alcohol (etanol) exhibe efectos bifásicos característicos, tanto en animales como en el hombre. Dosis bajas de la sustancia inducen estimulación locomotora en roedores, ${ }^{3}$ así como activación psicomotora y euforia en humanos, ${ }^{4}$ mientras que dosis altas disminuyen la actividad locomotora y producen sedación. ${ }^{5}$ En el cerebro y tejidos neuroendócrinos, el etanol modifica la actividad de numerosos neurotransmisores y neuromoduladores, incluyendo la dopamina (DA), la serotonina, el ácido gama aminobutírico (GABA), el glutamato, la acetilcolina, el neuropéptido $\mathrm{Y}$, el factor liberador de corticotropina (CRF) y los péptidos opioides. ${ }^{6-9}$ Estos sistemas neurales están implicados en forma diferencial en el reforzamiento positivo y la recompensa del etanol, así como en las conductas de alto consumo de la droga.

Evidencias bioquímicas y farmacológicas muestran que el sistema dopaminérgico (DAérgico) mesolímbico cumple un papel crucial en los mecanismos de reforzamiento y recom-

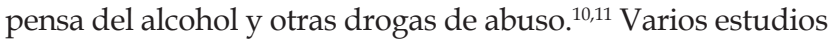
muestran que el etanol modula la transmisión DAérgica en el sistema mesolímbico y que la activación de esta vía conduce, eventualmente, al desarrollo de una conducta adictiva. El etanol aumenta la frecuencia de disparo de las neuronas DAérgicas en el área tegmental ventral (ATV) ${ }^{12}$ y la liberación y el metabolismo de dopamina (DA) en el núcleo accumbens (NAcc) y la corteza prefrontal. ${ }^{13,14}$ La liberación de DA en el NAcc inducida por etanol es un evento crítico en los mecanismos de reforzamiento y recompensa de las drogas de abuso.

Varios neurotransmisores y neuromoduladores, como los péptidos opioides (encefalinas, endorfinas y dinorfinas), modifican la actividad DAérgica del sistema mesocorticolímbico. Los agonistas del receptor opioide mu $(\mu)$, como la morfina, aumentan la frecuencia de disparo de las neuronas DAérgicas del $\mathrm{ATV}^{15}$ y estimulan la liberación y el metabolismo de DA en el NAcc y la corteza prefrontal, mientras que los agonistas del receptor opioide delta $(\delta)$ no tienen efecto. ${ }^{16-18}$ Estos hallazgos indican que la activación de los receptores opioides por opioides endógenos u opiáceos es crítica en la regulación de la transmisión DAérgica del sistema mesocorticolímbico. Además, estos estudios indican que los efectos de los péptidos opioides en esta vía son similares a los ejercidos por el etanol. De hecho, se ha mostrado que los opioides y el etanol comparten numerosas propiedades farmacológicas y exhiben efectos conductuales similares en animales y en el hombre. Por ejemplo, la administración de dosis bajas de etanol u opioides estimula la actividad locomotora a través de la activación DAérgica en el ATV, mientras que dosis altas activan terminales DAérgicas en el NAcc. ${ }^{19,20}$ La activación del sistema DAérgico mesocorticolímbico por agonistas $\mu$ y $\delta$ induce reforzamiento, mientras que la activación del receptor opioide kapa (к) está relacionada con la disforia. Estas acciones son mediadas por un aumento o una disminución en la liberación de DA en el NAcc, respectivamente. ${ }^{21}$ El conjunto de estos estudios sugiere que la dependencia a los opiáceos y al alcohol es mediada por un mecanismo neurobiológico común que implica la activación de circuitos DAérgicos de recompensa. ${ }^{11}$

\section{Participación de los péptidos opioides en el reforzamiento y la dependencia al alcohol}

Numerosas evidencias bioquímicas y farmacológicas sugieren que el reforzamiento positivo de alcohol y la conducta de alto consumo de la droga son mediados, al menos parcialmente, por un mecanismo que involucra la activación del sistema opioide endógeno inducida por etanol.,21,22 Esta activación aumentaría el valor hedónico y las propiedades reforzadoras del etanol, lo que a su vez mantendría una conducta 
de alto consumo de la sustancia. Asimismo, numerosas evidencias apoyan el papel central de los sistemas opioidérgicos en los mecanismos de reforzamiento del etanol y la conducta de alto consumo de la droga. La administración de dosis bajas de agonistas del receptor opioide $\mu$ aumenta el consumo de etanol en ratas, ${ }^{23-25}$ mientras que la de dosis altas lo disminuye. ${ }^{23,26}$ La administración de antagonistas no selectivos (naloxona y naltrexona) de los receptores opioides disminuye el consumo voluntario de etanol en roedores y monos. ${ }^{27-31}$ Los antagonistas selectivos de los receptores opioides $\mu$ y $\delta$ reducen también el consumo de etanol en distintos paradigmas experimentales. ${ }^{27,28,30,32-35}$ Por otra parte, estudios realizados en humanos muestran que la administración de naltrexona en pacientes alcohólicos reduce el comportamiento compulsivo por ingerir alcohol, así como la euforia y el número de recaídas. ${ }^{36,37} \mathrm{El}$ uso de ratones knockout ha permitido confirmar la participación de los receptores $\mu$ y $\delta$ en las conductas de consumo de etanol ${ }^{38,39} \mathrm{El}$ conjunto de estos estudios indica que la activación de los sistemas $\beta$-endorfinérgico y encefalinérgico vía los receptores $\mu$ y $\delta$ es relevante en el reforzamiento de etanol y en el mantenimiento de una conducta de alto consumo de la droga.

El uso de cepas y líneas de roedores seleccionadas genéticamente para preferir alcohol y consumir grandes cantidades de la droga ha contribuido a identificar los sistemas opioides específicos y los sustratos neurales implicados en el reforzamiento del etanol. Además, los estudios que utilizan manipulaciones farmacológicas de los sistemas opioidérgicos sobre el consumo de etanol han ayudado a investigar el papel funcional de los opioides en estos comportamientos. Sin embargo, son escasos los estudios que han investigado estos aspectos en animales no seleccionados genéticamente para preferir alcohol. Así, nuestro grupo ha estudiado los efectos de la administración aguda y crónica de etanol sobre distintos eventos de la transmisión de la Metionina-encefalina (Met-enk) y la $\beta$-endorfina, utilizando varios paradigmas experimentales en ratas Wistar. En particular, estudiamos los efectos del etanol sobre la expresión del ARNm de los precursores que dan origen a la Met-enk y a la $\beta$-endorfina (Pro-encefalina [Pro-enk] y Pro-opiomelanocortina [POMC]), así como sobre el contenido de los péptidos en regiones del sistema mesocorticolímbico y de la vía nigroestriatal. Además, investigamos los efectos de la administración aguda y crónica de etanol sobre la unión de ligandos selectivos de los receptores opioides $\mu$ y $\delta$ en esas áreas. Finalmente, investigamos si existe una correlación entre los cambios producidos por el etanol sobre la transmisión de estos péptidos y los efectos conductuales inducidos por la droga.

\section{Efectos agudos y crónicos del etanol sobre los sistemas encefalinérgico y $\beta$-endorfinérgico}

El etanol puede inducir alteraciones importantes sobre la biosíntesis, la liberación y la inactivación de los opioides, así como sobre la unión de opioides endógenos a sus receptores. Nuestros estudios iniciales se centraron en estudiar los efectos de la administración aguda de una dosis alta de etanol sobre la unión de ligandos selectivos de los recep-

Cuadro 1. Efecto de una dosis aguda de etanol sobre la unión de $\left[{ }^{3} \mathrm{H}\right]-\mathrm{DAMGO}$ al receptor opioide mu en regiones del sistema mesocorticolímbico y la vía nigroestriatal de la rata

\begin{tabular}{lcccr}
\hline & \multicolumn{4}{c}{ Tiempo después de la administración (h) } \\
\cline { 2 - 5 } Región & 0.5 & 1 & 2 & \multicolumn{1}{c}{4} \\
\hline Area tegmental ventral & $60.3 \pm 7.3 * * *$ & $83.0 \pm 8.2$ & $107.6 \pm 9.9$ & $103.9 \pm 6.1$ \\
Corteza prefrontal & $99.9 \pm 5.5$ & $86.4 \pm 3.8$ & $138.1 \pm 4.5 * * *$ & $108.1 \pm 3.2$ \\
Núcleo accumbens & & & & \\
• "core" & $102.0 \pm 4.2$ & $83.5 \pm 5.8$ & $106.7 \pm 5.5$ & $91.1 \pm 4.7$ \\
• "shell" & $99.4 \pm 3.3$ & $80.7 \pm 4.3 * *$ & $116.7 \pm 3.8$ & $92.2 \pm 2.5$ \\
Substantia nigra & & & & \\
- pars compacta & $11.8 \pm 5.6$ & $95.5 \pm 10.9$ & $100.0 \pm 11.1$ & $99.0 \pm 9.1$ \\
• pars reticulata & $142.1 \pm 10.2$ & $33.4 \pm 6.2 *$ & $79.1 \pm 17.9$ & $105.4 \pm 8.5$ \\
Caudado-putamen & & & & \\
1. Anterior-medial & & & & \\
• parches & & & & \\
• matriz & $98.9 \pm 5.3$ & $88.5 \pm 3.7$ & $103.0 \pm 3.5$ & $103.1 \pm 3.0$ \\
2. Medial-posterior & $106.2 \pm 4.5$ & $96.3 \pm 7.4$ & $72.7 \pm 9.0$ & $94.8 \pm 5.2$ \\
- parches & & & & \\
• matriz & $89.3 \pm 7.0$ & $85.9 \pm 7.2$ & $100.2 \pm 6.0$ & $82.5 \pm 6.0$ \\
3. Posterior & $87.8 \pm 8.3$ & $123.2 \pm 12.3$ & $79.6 \pm 24.2$ & $142.8 \pm 7.6$ \\
\hline
\end{tabular}

Ratas Wistar macho recibieron una dosis aguda de etanol $(2.5 \mathrm{~g} / \mathrm{kg})$ o agua (grupo control) por vía intragástrica y fueron sacrificados $30 \mathrm{~min}, 1,2 \circ 4 \mathrm{~h}$ después de la administración. La unión de [3H]-DAMGO $(8 \mathrm{nM})$ al receptor opioide mu se estudió por autoradiografía cuantitativa de receptores en cortes coronales de cerebro. Los datos se expresan como porciento del control en cada tiempo estudiado y son el promedio \pm EEM de 5 (control) o 6 (etanol) animales. *** $p<0.005 ;{ }^{* *} p<0.01 ;{ }^{*} p<0.05$, versus controles en cada tiempo (ANOVA de dos vías). 
Cuadro 2. Efecto de una dosis aguda de etanol sobre la unión de $\left[{ }^{3} \mathrm{H}\right]$-DPDPE al receptor opioide delta en regiones del sistema mesocorticolímbico y la vía nigroestriatal de la rata

\begin{tabular}{lcccc}
\hline & \multicolumn{4}{c}{ Tiempo después de la administración $(\mathrm{h})$} \\
\cline { 2 - 5 } Región & 0.5 & 1 & 2 & 4 \\
\hline $\begin{array}{l}\text { Corteza prefrontal } \\
\text { Núcleo accumbens } \\
\text { • "core" }\end{array}$ & $73.4 \pm 8.3$ & $119.4 \pm 13.9$ & $377.4 \pm 88.7^{* * *}$ & $100.5 \pm 6.5$ \\
• "shell" & $79.4 \pm 10.2$ & $121.0 \pm 14.3$ & $193.4 \pm 34.6^{* *}$ & $46.8 \pm 10.2$ \\
Substantia nigra & $90.0 \pm 9.9$ & $119.7 \pm 13.4$ & $197.1 \pm 37.1^{*}$ & $45.9 \pm 8.6$ \\
• Pars reticulata & $63.7 \pm 14.2$ & $266.6 \pm 32.2^{* * *}$ & $85.2 \pm 8.8$ & $70.0 \pm 17.3$ \\
Caudado-putamen & & & \\
$\begin{array}{l}\text { 1. Anterior-medial } \\
\text { • región lateral }\end{array}$ & $83.5 \pm 6.7$ & $139.6 \pm 9.2$ & $244.6 \pm 26.8^{* * *}$ & $93.9 \pm 10.7$ \\
2. Medial-posterior & $116.4 \pm 11.5$ & $167.3 \pm 11.1$ & $221.4 \pm 76.5^{* *}$ & $103.0 \pm 21.1$ \\
• región lateral & $147.3 \pm 11.1$ & $137.3 \pm 9.7$ & $296.1 \pm 65.5^{* * *}$ & $83.9 \pm 15.1$ \\
3. Posterior & $58.3 \pm 7.5 *$ & $133.1 \pm 6.6$ & $111.7 \pm 15.8$ & $121.3 \pm 11.2$ \\
\hline
\end{tabular}

Ratas Wistar macho recibieron una dosis aguda de etanol $(2.5 \mathrm{~g} / \mathrm{kg})$ o agua (grupo control) por vía intragástrica y fueron sacrificados $30 \mathrm{~min}, 1,2 \circ 4 \mathrm{~h}$ después de la administración. La unión de $\left[{ }^{3} \mathrm{H}\right]-\mathrm{DPDPE}(8 \mathrm{nM})$ al receptor opioide delta se estudió por autorradiografía cuantitativa de receptores en cortes coronales de cerebro. Los niveles de expresión del receptor delta en el área tegmental ventral y la substantia nigra pars compacta son muy bajos y no pudieron cuantificarse en este estudio. Los datos se expresan como porciento del control en cada tiempo estudiado y son el promedio \pm EEM de 5 (control; etanol 30 min y $2 \mathrm{~h}$ ) o 6 (etanol 1 y $4 \mathrm{~h}$ ) animales. ${ }^{* *} p<0.0001 ;{ }^{* *} p<0.01 ;{ }^{*} p<0.05$, versus controles en cada tiempo (ANOVA de dos vías).

tores $\mu$ y $\delta$ en áreas cerebrales asociadas a los circuitos de reforzamiento y recompensa de drogas de abuso. Estudiamos también los efectos agudos del etanol sobre la unión de estos ligandos en áreas de la vía nigroestriatal, ya que se ha propuesto que la actividad DAérgica de esta vía determina la sensibilidad cerebral al etanol. ${ }^{40}$ Encontramos que la administración de una dosis de etanol de $2.5 \mathrm{~g} / \mathrm{kg}$ en ratas Wistar altera la unión de ligandos selectivos de los receptores $\mu\left(\left[{ }^{3} \mathrm{H}\right]\left[\mathrm{D}-\mathrm{Ala}^{2}, \mathrm{MePhe}^{4}, \mathrm{Gly}-\mathrm{ol}^{5}\right]\right.$-encefalina) $\left(\left[{ }^{3} \mathrm{H}\right]-\mathrm{DA}\right.$ MGO) y $\delta\left(\left[{ }^{3} \mathrm{H}\right]\left[\right.\right.$ [D-Pen $\left.{ }^{2}, \mathrm{D}-\mathrm{Pen}^{5}\right]$-encefalina) ([$\left.\left.{ }^{3} \mathrm{H}\right]-\mathrm{DPDPE}\right)$ en forma diferencial en áreas discretas del cerebro de la rata, así como con distintos patrones cinéticos (cuadros 1 y 2). La administración aguda de etanol aumentó la unión de [ $\left.{ }^{3} \mathrm{H}\right]-$ DPDPE al receptor $\delta$ en la corteza prefrontal y el NAcc (core y shell) ${ }^{41}$ (cuadro 2), así como la unión de [ $\left.{ }^{3} \mathrm{H}\right]$-DAMGO al receptor $\mu$ en la corteza prefrontal ${ }^{42}$ (cuadro 1). El mismo tratamiento disminuyó la unión de $\left[{ }^{3} \mathrm{H}\right]$-DAMGO en el ATV y el shell del $\mathrm{NAcc}^{42}$ (cuadro 1). En la vía nigroestriatal, encontramos también diferencias importantes en cuanto a los efectos agudos del etanol y la cinética de respuesta (cuadros 1 y 2). La administración de la misma dosis de etanol disminuyó la unión de [ $\left.{ }^{3} \mathrm{H}\right]-\mathrm{DAMGO}$ (cuadro 1), pero aumentó la de $\left[{ }^{3} \mathrm{H}\right]-D P D P E$ (cuadro 2) en la substantia nigra, pars reticulata $(\mathrm{SNr}) \cdot{ }^{41,43}$ Este tratamiento aumentó también la unión de $\left[{ }^{3} \mathrm{H}\right]-\mathrm{DPDPE}$ en el caudado-putamen $(\mathrm{CP}),{ }^{41}$ pero no modificó la de $\left[{ }^{3} \mathrm{H}\right]$-DAMGO en esta región del cerebro. ${ }^{43}$

Los estudios descritos sugieren que las propiedades reforzadoras del etanol podrían ser parcialmente mediadas por mecanismos de regulación positiva y negativa de los receptores $\mu$ y $\delta$ en distintas áreas de las vías mesocorticolímbica y nigroestriatal (para revisiones, ver Méndez y Morales-Mulia, 2008a,b). ${ }^{44,45}$ De acuerdo con esta propuesta, los receptores $\mu$ en el ATV desempeñarían un papel crucial en la regulación de la actividad DAérgica del sistema mesocorticolímbico en respuesta a etanol, mientras que los receptores $\mu$ y $\delta$ tendrían un papel preponderante en el NAcc y la corteza prefrontal. En la vía nigroestriatal, ambos tipos de receptores cumplirían un papel importante a nivel de la substantia nigra (SN), pero sólo los receptores $\delta$ parecen estar implicados en la modulación DAérgica en el CP. Estos resultados indican que la modulación de la transmisión DAérgica en estas vías a través de la activación de receptores $\mu$ y $\delta$ ocurre por distintos mecanismos, y que la transmisión $\beta$-endorfinérgica y encefalinérgica desempeña un papel fundamental en el reforzamiento de etanol.

La siguiente etapa de nuestras investigaciones fue estudiar los efectos agudos del etanol sobre la expresión de Met-enk y $\beta$-endorfina en áreas cerebrales de los circuitos de reforzamiento y recompensa. La administración de una dosis alta de etanol $(2.5 \mathrm{~g} / \mathrm{kg})$ disminuyó la expresión del ARNm de Pro-enk en el ATV y la SN (pars compacta [SNc] y $\mathrm{SNr}$ ) de ratas Wistar, y la incrementó en la corteza prefrontal. $^{46,47}$ De manera interesante, este tratamiento produce un aumento prolongado y sostenido en los niveles del ARNm de Pro-enk en el NAcc, tanto en el core como en el shell, ${ }^{46}$ así como en distintas zonas del CP. ${ }^{47} \mathrm{La}$ administración de etanol también produce aumentos en la expresión de este ARNm en otras áreas cerebrales, como el núcleo paraventricular del hipotálamo, mientras que en otras, como el giro dentado y las regiones CA1, CA2 y CA3 del hipocampo, el etanol induce efectos bifásicos. ${ }^{48}$ Por otra parte, la administración de una dosis de etanol de $2.5 \mathrm{~g} / \mathrm{kg}$ disminuyó el contenido de Met-enk en el NAcc y el CP, pero no tuvo efecto en la corteza prefrontal. ${ }^{49}$ Dosis bajas o intermedias de etanol 
Péptidos opioides y mecanismos de reforzamiento y dependencia al alcohol

$(0.25,0.5$ y $1 \mathrm{~g} / \mathrm{kg})$ no modificaron el contenido del péptido en estas áreas del cerebro. ${ }^{49}$ En contraste con los efectos producidos por distintas dosis de etanol sobre el contenido de Met-enk en las regiones mencionadas, otros estudios de nuestro laboratorio revelaron que el etanol, en dosis intermedias y altas, estimula la liberación de Met-enk en el NAcc de la rata. ${ }^{49} \mathrm{El}$ efecto máximo se observó con una dosis de $1 \mathrm{~g} / \mathrm{kg}$ de etanol y la cinética de respuesta a la administración de etanol se presentó de manera diferencial. El conjunto de estos estudios indica que el sistema encefalinérgico es uno de los blancos importantes del etanol en el cerebro y la liberación de Met-enk inducida por etanol es uno de los eventos clave en esas acciones (para revisiones, ver Méndez y Morales-Mulia 2008b,c). ${ }^{45,50}$ Estos estudios sugieren, además, que las encefalinas desempeñan un papel crucial en el reforzamiento de etanol. La modulación de la transmisión DAérgica por opioides en el sistema mesocorticolímbico es, indudablemente, parte importante de este proceso.

De manera paralela a los estudios descritos, investigamos también los efectos de la administración de una sola dosis de etanol sobre la transmisión $\beta$-endorfinérgica. Sin embargo, la administración de una dosis de etanol de $2.5 \mathrm{~g} / \mathrm{kg}$ no modificó el contenido de $\beta$-endorfina en regiones como el ATV, la SN, el NAcc o la corteza prefrontal. ${ }^{51}$ En contraste, el mismo tratamiento disminuyó el contenido del péptido en el hipotálamo. Estos resultados sugieren que el sistema $\beta$-endorfinérgico participa selectivamente en los mecanismos de acción del etanol que están más asociados a los efectos neuroendócrinos de la droga. Estudios actualmente en curso en nuestro laboratorio confirmarán si éste es el caso. En particular, nuestro interés está dirigido a evaluar los cambios inducidos por la administración de etanol sobre la expresión del ARNm de POMC en distintas áreas del cerebro. Además del hipotálamo, regiones cerebrales de los circuitos de reforzamiento, como el ATV, el NAcc o la corteza prefrontal, contienen niveles muy bajos de este ARNm. ${ }^{52}$ Actualmente evaluamos si la exposición a una dosis alta de etanol modifica la expresión de este ARNm en la rata. Otros estudios de nuestro grupo, en colaboración con el de la doctora Bérod, muestran que la administración aguda de etanol induce la expresión de Fos en neuronas GABAérgicas y noGABAérgicas en el cerebro medio de la rata. ${ }^{53}$

Con el objeto de investigar si los efectos neuroquímicos del etanol sobre la transmisión opioidérgica se correlacionan con las acciones conductuales de la droga, llevamos a cabo una serie de experimentos en los que estudiamos el efecto de diferentes dosis de alcohol sobre la actividad motora en la rata. Observamos que dosis de etanol de $1 \mathrm{~g} / \mathrm{kg}$ aumentan el desplazamiento horizontal y las estereotipias, mientras que dosis altas de la sustancia $(2.5 \mathrm{~g} / \mathrm{kg})$ disminuyen estos parámetros y tienen un efecto sedante. ${ }^{54,55}$ Así, las alteraciones inducidas por dosis altas de alcohol sobre la transmisión de opioides en el cerebro previamente descritas podrían estar relacionadas con los efectos sedantes de la droga. Además, diversos factores pueden modificar de manera importante la respuesta motora a la administración de alcohol. En nuestro laboratorio hemos encontrado que factores como el ciclo circádico, el aislamiento o la exposición de los animales a ambientes novedosos tienen efectos muy importantes sobre la conducta locomotora de la rata. ${ }^{54-56}$ Estos y otros factores parecen ser determinantes en las conductas de alto consumo de alcohol y otras drogas de abuso.

Por otra parte, el análisis de los estudios de exposición crónica al alcohol reveló resultados interesantes. El tratamiento crónico con etanol $(10 \% \mathrm{v} / \mathrm{v}$, cuatro semanas) no modificó la unión de [ $\left.{ }^{3} \mathrm{H}\right]-\mathrm{DPDPE}$ en el ATV, la corteza prefrontal, el NAcc y la región anterior-medial del CP. ${ }^{57} \mathrm{Sin}$ embargo, observamos una tendencia de disminución en la unión del ligando en la SNc y la zona medial-posterior del $\mathrm{CP}$, resultado que actualmente está siendo confirmado en nuestro laboratorio. La unión de $\left[{ }^{3} \mathrm{H}\right]$-DAMGO al receptor $\mu$ en el ATV, la corteza prefrontal o el NAcc tampoco fue modificada por la exposición prolongada a etanol. ${ }^{51}$ Aunque estos estudios muestran que la exposición crónica a etanol por cuatro semanas no modifica la unión de los ligandos utilizados, queda por investigar si este tipo de tratamiento induce cambios en la funcionalidad de los receptores $\mu$ y $\delta$ en las regiones estudiadas.

En contraste con los datos obtenidos en el tratamiento agudo, la exposición crónica a etanol aumentó selectivamente el contenido de Met-enk en el ATV y la corteza prefrontal (cuadro 3), pero no modificó la concentración del péptido en el NAcc, el CP, la SN, el hipocampo y la amígdala. ${ }^{57} \mathrm{El}$ mismo tratamiento no modificó el contenido de $\beta$-endorfina en ninguna de estas áreas ${ }^{51}$ (cuadro 3). Estos resultados sugieren que las encefalinas mesocorticales participan en los cambios neuroadaptativos que ocurren durante la exposición prolongada a etanol.

Con el objeto de comparar los efectos crónicos de una droga de abuso (alcohol) con los producidos por reforzadores naturales como los azúcares, investigamos también el efecto de la exposición crónica a sacarosa en el mismo paradigma experimental. La exposición a sacarosa por cuatro semanas aumentó el contenido de Met-enk en el NAcc y el hipotálamo ${ }^{57}$ y el de $\beta$-endorfina en el NAcc y la $S^{51}$ (cuadro 3). La unión de $\left[{ }^{3} \mathrm{H}\right]$-DAMGO en el ATV, la corteza prefrontal y el NAcc no fue afectada por la sacarosa. En contraste, el azúcar aumentó la unión de [ $\left.{ }^{3} \mathrm{H}\right]-\mathrm{DPDPE}$ en la corteza prefrontal y en la región anterior-medial del CP. Además, observamos una tendencia de aumento en la unión de $\left[{ }^{3} \mathrm{H}\right]$-DPDPE en el NAcc (core y shell) y la SNr en los animales tratados crónicamente con sacarosa, efecto que está siendo confirmado en nuestro laboratorio. Estos resultados sugieren que las encefalinas y la $\beta$-endorfina presentes en el NAcc, la SN y el hipotálamo participan en los mecanismos que regulan la palatabilidad de sustancias.

El conjunto de estos estudios sugiere que la exposición prolongada a alcohol y/o sacarosa induce cambios selecti- 
Cuadro 3. Efecto de la exposición crónica a etanol y sacarosa sobre el contenido de Metionina-encefalina y $\beta$-endorfina en regiones del cerebro de la rata

\begin{tabular}{lcccc}
\hline & \multicolumn{3}{c}{ Etanol } & \multicolumn{2}{c}{ Sacarosa } \\
\cline { 2 - 5 } Región & Met-enk & $\beta$-endorfina & Met-enk & $\beta$-endorfina \\
\hline Area tegmental ventral & $\uparrow 25.3 \% * *$ & - & - & - \\
Corteza prefrontal & $\uparrow 245.0 \% * * *$ & - & - & - \\
Núcleo accumbens & - & - & $\uparrow 61.1 \% *$ & $\uparrow 100.0 \%$ * \\
Substantia nigra & - & - & - & $\uparrow 65.0 \% *$ \\
Caudado-putamen & - & & & \\
• anterior-medial & - & nd & - & nd \\
$\bullet$ medial-posterior & - & nd & - & nd \\
Hipotálamo & - & - & $\uparrow 55.8 \% *$ & - \\
\hline
\end{tabular}

Ratas Wistar macho fueron tratadas crónicamente con etanol $(10 \% \mathrm{v} / \mathrm{v})$, sacarosa (control isocalórico) o agua durante 4 semanas. El consumo de alimento y líquido fue pareado al del grupo de etanol y se incluyó además un grupo ad libitum con libre acceso al alimento y al agua. El contenido de los péptidos se determinó por radioinmunoensayo. Los datos se expresan como el porciento de cambio promedio con respecto al grupo ad libitum ( $n=8-10$ por grupo). Los cambios señalados con una flecha fueron significativos con respecto al grupo ad libitum (ANOVA de una vía). ${ }^{* \star *} p<0.001$; ${ }^{*}{ }^{*} \mathrm{p}<0.01 ;{ }^{*} \mathrm{p}<0.05 ; \mathrm{nd}=\mathrm{no}$ determinado; $-=\sin$ efecto.

vos en neuronas encefalinérgicas y/o $\beta$-endorfinérgicas en áreas discretas del cerebro de la rata. Los cambios observados en el sistema encefalinérgico podrían estar relacionados con los mecanismos de reforzamiento del alcohol y/o los azúcares, mientras que los encontrados en el $\beta$-endorfinérgico parecen estar más asociados al reforzamiento de los azúcares.

\section{CONCLUSIONES}

El conjunto de estudios realizados en nuestro laboratorio sugiere que la biosíntesis y liberación de péptidos opioides (Met-enk y $\beta$-endorfina), así como la activación de los receptores $\delta$ y $\mu$ representan eventos clave en la transmisión opioidérgica en las vías mesocorticolímbica y nigroestriatal sujetos a regulación por la exposición aguda y crónica de alcohol. Los efectos del etanol, además de ser dependientes de la dosis y la duración de la exposición a la droga, son región-específicos. Los cambios observados en los sistemas encefalinérgico y $\beta$-endorfinérgico en los animales tratados crónicamente con etanol probablemente forman parte de las neuroadaptaciones que se establecen progresivamente en el cerebro durante la exposición prolongada a la droga. Finalmente, nuestros resultados sugieren que estos sistemas peptidérgicos participan en los mecanismos de reforzamiento del alcohol y/o los azúcares. En particular, el sistema encefalinérgico parece estar implicado en el reforzamiento de alcohol y azúcares, mientras que el $\beta$-endorfinérgico parece estar más asociado al reforzamiento de azúcares. Queda por determinar si durante la privación de alcohol y/o sacarosa (abstinencia) ocurren cambios específicos en estos sistemas peptidérgicos en las regiones estudiadas, así como la relevancia de los mismos en los mecanismos de dependencia a estas sustancias.

\section{AGRADECIMIENTOS}

Mi agradecimiento y reconocimiento a Mariana Leriche Vázquez, Fabiola Hernández Vázquez e Irais G. Barbosa-Luna, estudiantes de mi laboratorio, que desarrollaron una parte importante de las investigaciones discutidas en este trabajo. Los estudios presentados fueron financiados por el Consejo Nacional de Ciencia y Tecnología (CONACyT) (3261P-N9607, 34359-N y 82728), por ANUIES/CONACyT/ECOS-Nord (M01S02) y por el Instituto de Ciencia y Tecnología del Distrito Federal (ICyTDF PICDS08-13).

\section{REFERENCIAS}

1. Berruecos L. La investigación psicosocial y cultural acerca del consumo de alcohol y el alcoholismo en México. Psiquis 2002;11:39-45.

2. Instituto Nacional de Psiquiatría Ramón de la Fuente Muñiz, Secretaría de Salud. Encuesta Nacional de Adicciones 2011: Reporte de alcohol. México, DF: 2011; pp 92.

3. Carlsson A, Engel J, Svensson TH. Inhibition of ethanol-induced excitation in mice and rats by -methyl-p-tyrosine. Psychopharmacologia 1972;26:307-312.

4. Martin CS, Earlywine M, Musty RE, Perrine MW et al. Development and validation of the Biphasic Alcohol Effects Scale. Alcohol Clin Exp Res 1993;17:140-146.

5. Earleywine M. Confirming the factor structure of the anticipated biphasic alcohol effects scale. Alcohol Clin Exp Res 1994;18:861-866.

6. Ulm RR, Volpicelli JR, Volpicelli LA. Opiates and alcohol self-administration in animals. J Clin Psychiatry 1995;56(Supl 7):5-14.

7. Overstreet DH. Behavioral characteristics of rat lines selected for differential hypothermic responses to cholinergic or serotonergic agonists. Behav Genet 2002;32:335-348.

8. Cowen MS, Lawrence AJ. Alcoholism and neuropeptides: an update. CNS Neurol Disord Drug Targets 2006;5:233-239.

9. Crabbe JC, Phillips TJ, Harris RA, Arends MA et al. Alcohol-related genes: contributions from studies with genetically engineered mice. Addict Biol 2006;11:195-269.

10. Koob GF, Sanna PP, Bloom FE. Neuroscience of addiction. Neuron 1998;21:467-476.

11. Wise RA, Bozarth MA. A psychomotor stimulant theory of addiction. Psychol Rev 1987;94:469-492. 
12. Gessa GL, Muntoni F, Collu M, Vargiu L et al. Low doses of ethanol activate dopaminergic neurons in the ventral tegmental area. Brain Res 1985;348:201-203.

13. Fadda F, Argiolas A, Melis MR, Serra G et al. Differential effects of acute and chronic ethanol on dopamine metabolism in frontal cortex, caudate nucleus and substantia nigra. Life Sci 1980;27:979-986.

14. Di Chiara G, Imperato A. Ethanol preferentially stimulates dopamine release in the nucleus accumbens of freely moving rats. Eur J Pharmacol 1985;115:131-132.

15. Gysling K, Wang RY. Morphine-induced activation of A10 dopamine neurons in the rat. Brain Res 1983;277:119-127.

16. Heijna MH, Padt M, Hogenboom F, Portoghese PS et al. Opioid receptor-mediated inhibition of dopamine and acetylcholine release from slices of rat nucleus accumbens, olfactory tubercle and frontal cortex. Eur J Pharmacol 1990;181:267-278.

17. Spanagel R, Herz A, Shippenberg TS. Identification of the opioid receptor types mediating beta-endorphin-induced alterations in dopamine release in the nucleus accumbens. Eur J Pharmacol 1990;190:177-184.

18. Wood PL, Rao TS. Morphine stimulation of mesolimbic and mesocortical but not nigrostriatal dopamine release in the rat as reflected by changes in 3-methoxytyramine levels. Neuropharmacology 1991;30:399-401.

19. Joyce EM, Iversen SD. The effect of morphine applied locally to mesencephalic dopamine cell bodies on spontaneous motor activity in the rat. Neurosci Lett 1979;14:207-212.

20. Kalivas PW, Widerläv E, Stanley D, Breese G et al. Enkephalin action on the mesolimbic system: a dopamine-dependent and a dopamineindependent increase in locomotor activity. J Pharmacol Exp Ther 1983;227:229-237.

21. Herz A. Endogenous opioid systems and alcohol addiction. Psychopharmacology (Berl) 1997;12:99-111.

22. Froehlich JC. Genetic factors in alcohol self-administration. J Clin Psychiatry 1995;56(Supl 7):15-23.

23. Reid LD, Czirr SA, Bensinger CC, Hubbell CL et al. Morphine and diprenorphine together potentiate intake of alcoholic beverages. Alcohol 1987;4:161-168.

24. Wild KD, Reid LD. Modulation of ethanol-intake by morphine: evidence for a central site of action. Life Sci 1990;47:PL49-PL54.

25. Stromberg MF, Meister SC, Volpicelli JR, Ulm RR. Low dose of morphine and the consumption of a sweetened ethanol solution: differential effects on acquisition and maintenance. Alcohol 1997;14:463-468.

26. Volpicelli JR, Ulm RR, Hopson N. Alcohol drinking in rats during and following morphine injections. Alcohol 1991;8:289-292.

27. Lê AD, Poulos CX, Quan B, Chow S. The effects of selective blockade of delta and mu opiate receptors on ethanol consumption by C57BL/6 mice in a restricted access paradigm. Brain Res 1993;630:330-332.

28. Franck J, Lindholm S, Raaschou P. Modulation of volitional ethanol intake in the rat by central delta-opioid receptors. Alcohol Clin Exp Res 1998;22:1185-1189.

29. Stromberg MF. The effect of baclofen alone and in combination with naltrexone on ethanol consumption in the rat. Pharmacol Biochem Behav 2004;78:743-750.

30. Higley AE, Kiefer SW. Delta receptor antagonism, ethanol taste reactivity, and ethanol consumption in outbred male rats. Alcohol 2006;40:143-150.

31. Stromberg MF, Volpicelli JR, O'Brien CP. Effects of naltrexone administered repeatedly across 30 or 60 days on ethanol consumption using a limited access procedure in the rat. Alcohol Clin Exp Res 1998;22:2186-2191.

32. Krishnan-Sarin S, Wand GS, Li XW, Portoghese PS et al. Effect of mu opioid receptor blockade on alcohol intake in rats bred for high alcohol drinking. Pharmacol Biochem Behav 1998;59:627-635.

33. Krishnan-Sarin S, Jing SL, Kurtz DL, Zweifel M et al. The delta opioid receptor antagonist naltrindole attenuates both alcohol and saccharin intake in rats selectively bred for alcohol preference. Psychopharmacology 1995;120:177-185.
34. Krishnan-Sarin S, Portoghese PS, Li TK, Froehlich JC. The delta 2opioid receptor antagonist naltriben selectively attenuates alcohol intake in rats bred for alcohol preference. Pharmacol Biochem Behav 1995;52:153-159.

35. Myers RD, Borg S, Mossberg R. Antagonism by naltrexone of voluntary alcohol selection in the chronically drinking macaque monkey. Alcohol 1986;3:383-388.

36. O'Malley S, Jaffe AJ, Chang G, Schottenfeld RS et al. Naltrexone and coping skills therapy for alcohol dependence. Arch Gen Psychiatry 1992;49:881-887.

37. Volpicelli JR, Alterman AI, Hayashida M, O'Brien CP. Naltrexone in the treatment of alcohol dependence. Arch Gen Psychiatry 1992;49:876-880.

38. Roberts AJ, McDonald JS, Heyser CJ, Kieffer BL et al. mu-Opioid receptor knockout mice do not self-administer alcohol. J Pharmacol Exp Ther 2000;293:1002-1008.

39. Roberts AJ, Gold LH, Polis I, McDonald JS et al. Increased ethanol self-administration in delta-opioid receptor knockout mice. Alcohol Clin Exp Res 2001;25:1249-1256.

40. Yanai J, Shaanani R, Pick CG. Altered brain sensitivity to ethanol in mice after MPTP treatment. Alcohol 1995;12:127-130.

41. Méndez M, Morales-Mulia M, Leriche M. [3H]DPDPE binding to $\delta$ opioid receptors in the rat mesocorticolimbic and nigrostriatal pathways is transiently increased by acute ethanol administration. Brain Res 2004;1028:180-190.

42. Méndez M, Leriche M, Calva JC. Acute ethanol administration differentially modulates $\mu$ opioid receptors in the rat meso-accumbens and mesocortical pathways. Mol Brain Res 2001;94:148-156.

43. Méndez M, Leriche M, Calva JC. Acute ethanol administration transiently decreases $\left[{ }^{3} \mathrm{H}\right]$-DAMGO binding to mu opioid receptors in the rat substantia nigra pars reticulata but not in the caudate-putamen. Neurosci Res 2003;47:153-160.

44. Méndez M, Morales-Mulia M. Role of mu and delta opioid receptors in alcohol drinking behaviour. Current Drug Abuse Reviews 2008;1:239-252.

45. Méndez M, Morales-Mulia M. Role of endogenous opioid peptides in ethanol reinforcement. En: Méndez M, Mondragón-Ceballos R (eds). Neural mechanisms of action of drugs of abuse and natural reinforcers. Kerala, India: Research Signpost; 2008; pp 41-60.

46. Méndez M, Morales-Mulia M. Ethanol exposure differentially alters pro-enkephalin mRNA expression in regions of the mesocorticolimbic system. Psychopharmacology 2006;189:117-124.

47. Méndez M, Morales-Mulia M, Pérez-Luna JM. Ethanol-induced changes in Proenkephalin mRNA expression in the rat nigrostriatal pathway. J Mol Neurosci 2008;34:225-234.

48. de Gortari P, Méndez M, Rodríguez-Keller I, Pérez-Martínez L et al. Acute ethanol administration induces changes in TRH and proenkephalin expression in hypothalamic and limbic regions of rat brain. Neurochem Int 2000;37:483-496.

49. Méndez M, Barbosa-Luna IG, Pérez-Luna JM, Cupo A et al. Effects of acute ethanol administration on Methionine-enkephalin expression and release in regions of the rat brain. Neuropeptides 2010;44:413420.

50. Méndez M, Morales-Mulia M. Ethanol exposure and Pro-enkephalin mRNA expression in regions of the mesocorticolimbic system. En: Sher L (ed). Research on the neurobiology of alcohol use disorders. New York: Nova Science Publishers, Inc.; 2008; pp 177-202.

51. Leriche M, Méndez $M$. Ethanol exposure selectively alters $\beta$-endorphin content but not $\left[{ }^{3} \mathrm{H}\right]$-DAMGO binding in discrete regions of the rat brain. Neuropeptides 2010;44:9-16.

52. Leriche M, Cote-Vélez A, Méndez M. Presence of Pro-opiomelanocortin mRNA in the rat medial prefrontal cortex, nucleus accumbens and ventral tegmental area: studies by RT-PCR and in situ hybridization techniques. Neuropeptides 2007;41:421-431.

53. Leriche M, Méndez M, Zimmer L, Bérod A. Acute ethanol induces Fos in GABAergic and non GABAergic neurons of the forebrain : a double 
labeling study in the medial prefrontal cortex and extended amygdala. Neuroscience 2008;153:259-267.

54. Hernández-Vázquez F. Efectos estimulantes y depresores del alcohol sobre la actividad locomotora en ratas Wistar. Tesis de licenciatura en Biología, Facultad de Ciencias, UNAM, México; 2005.

55. Hernández-Vázquez F, Méndez M. Stimulant and depressant behavioral actions of alcohol in non-selected Wistar rats. En: Chen FJ (ed). New trends in brain research. New York: Nova Science Publishers, Inc.; 2006; pp. 127-142.
56. Hernández-Vázquez F. Sensibilización conductual al alcohol en ratas Wistar. Tesis de maestría en Ciencias Biológicas (Biología experimental), Facultad de Medicina, UNAM, México; 2009.

57. Barbosa-Luna IG. Participación de las encefalinas en los mecanismos de neuroadaptación al alcohol en la vía mesocorticolímbica de la rata. Tesis de maestría en Ciencias Biológicas (Biología experimental), Facultad de Medicina, UNAM, México; 2012.

Artículo sin conflicto de intereses 